\title{
A Novel Non-integer Indirect Adaptive Control for Non-integer Order Systems with Non-prior Knowledge
}

\author{
Bachir Bourouba \\ Department of Electrical Engineering \\ Ferhat Abbas Setif-1 University \\ Setif, Algeria \\ bourouba_b@yahoo.fr
}

\author{
Samir Ladaci \\ Department of Electronics, Electrical Engineering and \\ Automation, National Polytechnic School of Constantine \\ Constantine, Algeria \\ samir_ladaci@yahoo.fr
}

\begin{abstract}
In this study, a new non-integer indirect adaptive control method with reference model is suggested for the class of non-integer order systems. The objective of model reference control is to include the output of the given reference fractional model in tracking the output of a controlled plant by using the concept of on-line goal adaptation. The stability of the closed-loop system is analyzed via the Lyapunov method. Finally, Matlab simulation results are presented to illustrate the effectiveness of the proposed method of indirect fractional model reference adaptive control.
\end{abstract}

Keywords-non integer order system; fractional adaptive control; MRAC; control system; Lyapunov stability

\section{INTRODUCTION}

Model reference adaptive control (MRAC) is one of the main approaches of adaptive control. The fundamental goal of adaptive control is to treat with system uncertainty and/or time varying system parameters. The essential idea in adaptive control is to design a controller which adapts itself to plant uncertainty or time variance in the plant dynamics. Adaptive control is one of the most important topics in the literature on control when the model of the system is uncertain [1-7]. Fundamental research efforts have been focused on MRAC designs for linear plants with parametric uncertainty. Generally in model reference adaptive control, the goal is to have a system that follows a certain system as a reference model. In reality, an adaptive controller is designed to achieve this goal, despite the uncertainties that may occur in the parameters of the system [8]. Fractional-order calculus is an area of mathematics that deals with integrals and derivatives of non-integer orders. In other words, it is a generalization of the traditional calculus that leads to similar concepts and tools, but with a much wider applicability. In the last three decades, fractional calculus has been rediscovered by engineers and scientists. It is applied in an increasing number of fields, including control theory among them. As an example, the modeling of viscoelastic materials has been done in many old and recent works using fractional order derivatives [10-11]. The success of non-integer order controllers is unquestionable due to the emergence of effective methods in differentiation and integration of non-integer order equations [12-13]. In recent years, an increasing number of studies related with the application of the fractional calculus (FC) theory are observed in many areas of science and engineering [14].

In this paper, a new control structure named Non-integer Indirect Adaptive Control for a Class of Non-integer Order System with Non-prior Knowledge is proposed to investigate the control for a class of fractional system. This novel method of control is based in MRAC configuration. Based on already reported results, in this paper, we propose a novel method to control a class of linear fractional-order systems based in MRAC configuration. This novel methodology of non-integer indirect adaptive control with reference model is proposed to guarantee stability and the tracking of the reference model. Two simulation examples are given to illustrate the performance obtained by the proposed indirect adaptive fractional control scheme.

\section{FRACTIONAL-ORDER DEFINITION AND PRELIMINARIES}

Fractional-order calculus or non-integer-order calculus is the generalization of the classical integer order calculus. There are several definitions for fractional order derivative and integral. Fractional calculus is considered as an extension of integer-order calculus to non-integer order calculus. The theory of derivatives and integrals of non-integer order was firstly mentioned by Leibnitz. After that, other definitions were generated by Liouville, Grunwald, Letnikov and Riemann. The integro-differential operator is given by ${ }_{a} D_{t}^{\alpha}$ where:

$$
{ }_{a} D_{t}^{\alpha}= \begin{cases}\frac{d^{\alpha}}{d t^{\alpha}} & \operatorname{Re}(\alpha)>0 \\ 1 & \operatorname{Re}(\alpha)=0 \\ \int_{a}^{t} d \tau^{(-\alpha)} & \operatorname{Re}(\alpha)<0\end{cases}
$$

where $a$ denotes lower limits and $t$ are the upper limits respectively of the operator, and $a \in \mathrm{R}$ is the order of integration or differentiation $[9,15,16]$. 
The Riemann-Liouville derivative definition of the order $\alpha$ is described as:

$$
{ }_{a} D_{t}^{\alpha} g(t)=\frac{d^{n}}{d t^{n}}\left[\frac{1}{\Gamma(n-\alpha)} \int_{a}^{t} \frac{g(\tau)}{(t-\tau)^{\alpha-n+1}} d \tau\right]
$$

where $n-1<\alpha<n, n \in \mathbb{N}$ and $\Gamma(\alpha)=\int_{0}^{\infty} x^{\alpha-1} e^{-x}$ is the Gamma function, and $f(t)$ is a continuous time function.

The following definition is given in $[13,14]$ :

$$
{ }_{a} D_{t}^{\alpha} g(t)=\frac{1}{\Gamma(n-\alpha)} \int_{a}^{t} \frac{g^{(n)}(\tau)}{(t-\tau)^{\alpha-n+1}} d \tau
$$

The Grunwald-Letnikov's derivative definition can be written as $[15,16]$ :

$$
\begin{array}{r}
D^{\alpha} g(t)=\lim _{h \rightarrow 0} \frac{1}{h^{\alpha}} \sum_{j=0}^{\left[\frac{t-a}{h}\right]}(-1)^{k}\left(\begin{array}{c}
\alpha \\
j
\end{array}\right) g(t-j h) \\
\left(\begin{array}{c}
\alpha \\
j
\end{array}\right)=\frac{\alpha !}{j !(\alpha-j) !}
\end{array}
$$

where $h$ denotes the time increment, $\left[\frac{t-a}{h}\right]$ is a flooring operator and the binomial coefficients.

As the Grunwald-Letnikov's fractional operator is more consistent than others, then the Grunwald-Letnikov's fractional-order differential equations operator will be employed in this paper for computer numerical simulations due to its wide applications in engineering and well-understood physical interpretation. If the initial conditions are taken as zero, the Laplace transform of differintegral operator ${ }_{a} D_{t}^{\alpha} g(t)$ is obtained as:

$$
L\left\{{ }_{a} D_{t}^{\alpha} g(t)\right\}=L\left\{\frac{d^{\alpha} g(t)}{d t^{\alpha}}\right\}=s^{\alpha} L\{g(t)\}
$$

In general, any fractional-order single input single output (SISO) system can be described by a fractional differential equation of the form $[9,11]$ :

$$
\begin{aligned}
& a_{n} D_{t}^{\alpha_{n}} y(t)+a_{n-1} D_{t}^{\alpha_{n-1}} y(t)+\ldots .+a_{0} D_{t}^{\alpha_{0}} y(t) \\
& =b_{m} D_{t}^{\beta_{m}} u(t)+b_{m-1} D_{t}^{\beta_{m-1}} u(t)+\ldots+b_{0} D_{t}^{\beta_{0}} u(t)
\end{aligned}
$$

where

$$
\left\{\begin{array}{l}
\alpha_{0}<\alpha_{1},<\alpha_{2}<\ldots<\alpha_{n} \\
\beta_{0}<\beta_{1}<\beta_{2}<\ldots<\beta_{m}
\end{array}\right.
$$

indicates the fractional-orders and $a_{0}, a_{1}, a_{2}, \ldots, a_{n} b_{0}, b_{1}, \ldots, b_{m}$ denote the system parameters.

If $\alpha_{0},=\alpha_{1}=\alpha=\ldots=\alpha_{n}$ and $\beta_{0}=\beta_{1}=\ldots=\beta_{m}$, the system (8) is called a commensurate order system, otherwise system (6) indicates an incommensurate order system.

\section{MODEL REFERENCE ADAPTIVE FRACTIONAL CONTROL}

Adaptive control is a well-researched topic in control theory spanning several decades in research. The model reference adaptive control system is an important scheme in adaptive control. It may be treated as an adaptive system in which the desired performance is expressed in terms of a reference model. The configuration of the model reference adaptive control block is given in Figure 1. In the configuration MRAC the desired behavior is specified by a reference model, and the parameters of the controller are adjusted based on the error, which is the difference between the outputs of the closedloop system and the outputs of the reference model. The mechanism for adjusting the parameters of the controller in a model reference adaptive control can be obtained by applying the stability theory of Lyapunov. The MRAC structure is divided in four main parts: the plant, the controller, the reference model and the adjustment mechanism.

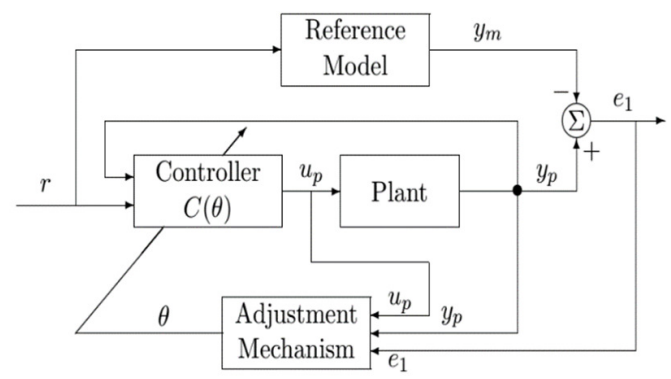

Fig. 1. Block diagram scheme of adaptive control with reference model

To introduce the model reference fractional adaptive control problem, we consider a class of commensurate fractional order systems formulated as:

$$
D_{t}^{q} y(t)=a \cdot y(t)+b \cdot u(t)
$$

where $x(t) \in \mathbb{R}$ and $u(t) \in \mathbb{R}$ are the system state and the control variable respectively, $a$ and $b$ are unknown constant parameters but $\operatorname{sgn}(b)$ is known. The fractional reference model was chosen to generate the desired trajectory $y_{m}(t)$ which the plant output $y(t)$ has to flow. The reference model is given by:

$$
D_{t}^{q} y_{m}(t)=a_{m} \cdot y_{m}(t)+b_{m} \cdot r(t)
$$

Subject to $y_{m}(0)=y_{m 0}$, the reference model is stable, i.e., $a_{m}<0$ and $r(t)$ is the reference input. The parameters $a_{m}$ and $b_{m}$ are known constants, $y_{m}(t)$ and $r(t)$ are measured at each time $t$. The design objective is to make the tracking error $e(t)=y(t)-y_{m}(t)$ converge to 0 . Let us first design a Model Reference Control (MRC), that is, the control design assuming all the parameters are known, to ensure that the output $y(t)$ follows $y_{m}(t)$. We express the closed-loop system in terms of the time derivative of the tracking error

$$
\begin{aligned}
e^{(q)}(t) & =y^{(q)}(t)-y^{(q)}(t) \\
& =a_{m} e(t)+b\left[u(t)-\frac{a_{m}-a}{b} y(t)-\frac{b_{m}}{b} r(t)\right]
\end{aligned}
$$


The basic state-feedback state tracking problem of this section is to design a state feedback law $u(t)$ to control the plant given by (7). Firstly, we define an ideal controller that perfectly cancels out the uncertainty and enables $x(t)$ as:

$$
u(t)=\psi_{1}^{*} \cdot y(t)+\psi_{2}^{*} \cdot r(t)
$$

where

$$
\psi_{1}^{*}=\frac{a_{m}-a}{b} \text { and } \psi_{2}^{*}=\frac{b_{m}}{b}
$$

The superscript $(*)$ denotes ideal constant values which are unknown. The tracking error equation is established as:

$$
e^{(q)}(t)-a_{m} e(t)=0
$$

The tracking error converges to zero exponentially and the system is asymptotically stable. When the plant parameters $a, b$ are unknown, (10) cannot be implemented. To solve the control problem, we develop a new fractional indirect adaptive control design, which first adaptively updates the estimates of the plant parameters $a$ and $b$ and then calculates the controller parameters $\psi_{1}(t)$ and $\psi_{2}(t)$ in (10) from the plant parameter estimates. The real control input is:

$$
u(t)=\psi_{1}(t) \cdot y(t)+\psi_{2}(t) \cdot r(t)
$$

where:

$$
\begin{aligned}
& \psi_{1}(t)=\frac{a_{m}-\hat{a}(t)}{\hat{b}(t)} \\
& \psi_{2}(t)=\frac{b_{m}}{\hat{b}(t)}
\end{aligned}
$$

$\hat{a}(t)$ and $\hat{b}(t)$ are the estimates of $a$ and $b$ respectively, at time $t$, and search for an adaptive law to generate $\hat{a}(t)$ and $\hat{b}(t)$ online.

Let $\tilde{a}=\hat{a}(t)-a$ and $\tilde{b}=\hat{b}(t)-b$ be the estimation errors. Now, the plant model (7) is expressed as:

$$
D_{t}^{(q)} y(t)=a \cdot y(t)+(\hat{b}(t)-\tilde{b}) \cdot u(t)
$$

Substituting (15), (16) and (17) into (18) we get:

$$
\begin{aligned}
& D_{t}^{(q)} y(t)=a \cdot y(t)+(\hat{b}(t)-\tilde{b}) \cdot\left[\frac{a_{m}-\hat{a}(t)}{\hat{b}(t)} y(t)+\frac{b_{m}}{\hat{b}(t)} r(t)\right] \\
& =\left[\begin{array}{r}
a \cdot y(t)+\hat{b}(t) \cdot\left[\frac{a_{m}-\hat{a}(t)}{\hat{b}(t)} y(t)+\frac{b_{m}}{\hat{b}(t)} r(t)\right] \\
-\tilde{b} \cdot\left[\frac{a_{m}-\hat{a}(t)}{\hat{b}(t)} y(t)+\frac{b_{m}}{\hat{b}(t)} r(t)\right]
\end{array}\right] \\
& =\left(a_{m}-\tilde{a}\right) y(t)+b_{m} r(t)-\tilde{b} \cdot\left[\frac{a_{m}-\hat{a}(t)}{\hat{b}(t)} y(t)+\frac{b_{m}}{\hat{b}(t)} r(t)\right]
\end{aligned}
$$

Based in the tracking error $e(t)=y(t)-y_{m}(t)$, from (17) and (7) we have:

$$
e^{(q)}(t)=a_{m} e(t)-\tilde{a} y(t)-\tilde{b}\left[\frac{a_{m}-\hat{a}(t)}{\hat{b}(t)} y(t)+\frac{b_{m}}{\hat{b}(t)} r(t)\right]
$$

Lemma 1: assume $f(t) \in \mathbb{R}$ is a continuous and derivable function. Then, for any time instant $t \geq t_{0}$ the following inequality holds $[12,17,18]$ :

$$
\frac{1}{2}{ }_{t_{0}}^{C} D_{t}^{\alpha} f^{2}(t) \leq f(t)_{t_{0}}^{C} D_{t}^{\alpha} f(t)
$$

Stability proof. In order to analyze the closed-loop stability and to find the adaptive laws we consider the following measure for the error $e(t), \tilde{a}(t)$, and $\tilde{b}(t)$.

$$
V(e, \tilde{a}, \tilde{b})=\frac{1}{2} e^{2}+\frac{1}{2 \varphi_{1}} \tilde{a}^{2}+\frac{1}{2 \varphi_{2}} \tilde{b}^{2}
$$

The positive real design parameters $\varphi_{1}>0$ and $\varphi_{2}>0$ are often referred to as adaptive gains, as they can affect the speed of parameter adaptation. Taking fractional derivative of (22) with respect to time and using Lemma 1, we have:

$$
\begin{gathered}
V^{(q)} \leq e e^{(q)}+\frac{1}{\varphi_{1}} \tilde{a}^{(q)} \tilde{a}+\frac{1}{\varphi_{2}} \tilde{b}^{(q)} \tilde{b} \\
\leq\left[\begin{array}{l}
a_{m} e^{2}-\tilde{a}\left[\frac{\tilde{a}^{(\alpha)}}{\varphi_{1}}+e y\right]+ \\
\tilde{b}\left[\frac{\tilde{b}^{(q)}}{\varphi_{2}}-\left[\frac{a_{m}-\hat{a}}{\hat{b}} y(t)+\frac{b_{m}}{\hat{b}} r\right] e\right]
\end{array}\right] \\
V^{(q)} \leq\left[\begin{array}{l}
e\left[a_{m} e-\tilde{a} y-\tilde{b}\left[\frac{a_{m}-\hat{a}}{\hat{b}} y(t)+\frac{b_{m}}{\hat{b}} r\right]\right] \\
+\frac{1}{\varphi_{1}} \tilde{a}^{(q)} \tilde{a}+\frac{1}{\varphi_{2}} \tilde{b}^{(q)} \tilde{b}
\end{array}\right]
\end{gathered}
$$

Note that $\dot{\tilde{a}}=\dot{\hat{a}}(t)$ and $\dot{\tilde{b}}=\dot{\hat{b}}(t)$. Based on the updated estimates of $\hat{a}(t)$ and $\hat{b}(t)$, we can calculate the parameters:

$$
\left\{\begin{array}{l}
\hat{a}^{(q)}(t)=-\varphi_{1} \cdot e(t) \cdot y(t) \\
\hat{b}^{(q)}(t)=-\varphi_{2}\left[\frac{a_{m}-\hat{a}(t)}{\hat{b}(t)} y(t)+\frac{b_{m}}{\hat{b}(t)} r(t)\right] e(t)
\end{array}\right.
$$

From (22) and (24) we have:

$$
V^{(q)} \leq a_{m} e^{2}
$$

This implies that $V(e, \tilde{a}, \tilde{b})$ as a function of $t$ does not increase, that $e(t), \tilde{a}(t)$, and $\tilde{b}(t)$ are bounded and so is $u(t)$ in (18). Then, from (25), $\int_{0}^{\infty} e^{2}(t) d t<\infty$, and $e^{(q)}(t)$ in (17), is $e(t) \in L^{2}$. Finally, by using the lemma of Barbalat's given in $[5,15-17]$ we conclude that the system is Lyapunov stable and 
the tracking error $\quad \lim _{t \rightarrow \infty}\left[y(t)-y_{m}(t)\right]=0 \quad$ converges asymptotically to zero.

\section{NUMERICAL SIMULATIONS}

In this section, some numerical simulation studies are carried out to show the effectiveness of the proposed control scheme to the non-integer order system.

Firstly, let us consider an unstable non integer order system defined by:

$$
D_{t}^{q} y(t)=y(t)+2 . u(t)
$$

To build the adaptive controller we choose a model reference given by :

$$
D_{t}^{q} y_{m}(t)=-y_{m}(t)+r(t)
$$

where $q=0.98$.

The values of the parameters of adaptive gain were chosen as: $\varphi_{1}=20$ and $\varphi_{2}=80$. The control law necessitates the reference model, which has two design parameters $a_{m}$ and $b_{m}$. Both of them can be chosen without restraint, nevertheless it is worth to set $a_{m}=1$ and $b_{m}=1$ to get a 0.78 gain of reference dynamics. The initial conditions of the system and reference model are $y(0)=0$ and $y_{m}(0)=0$. The reference input signal $r(t)$ is given by:

$$
r(t)=\cos (2 \pi t)
$$

If the parameters of the system and reference model are known, the control law can be calculated as:

$$
u(t)=\psi_{1}^{*} \cdot y(t)+\psi_{2}^{*} \cdot r(t)
$$

From (10) we obtain:

$$
\psi_{1}^{*}=\frac{a_{m}-a}{b}=-2
$$

and:

$$
\psi_{2}^{*}=\frac{b_{m}}{b}=1
$$

The simulation results for this case are shown in Figures 23 , where we show the time responses of the plant state $y(t)$ and the output of the reference model $y_{m}(t)$ and the control $u(t)$.

In the case where the system parameters are known, we can calculate the parameters of the controller directly, and from simulation the output of the system $y(t)$ converges quickly to the output of the reference model $y_{m}(t)$. When the parameters of the system are unknown, $a$ and $b$ are estimated by $\hat{a}(t)$ and $\hat{b}(t)$ respectively. To determine the controller parameter gains $\psi_{1}(t), \psi_{2}(t),(14)$ and (15) are used. The on-line estimate is generated by the adaptive law (24). The control law, based on the certainty equivalence principle, is given by (13).

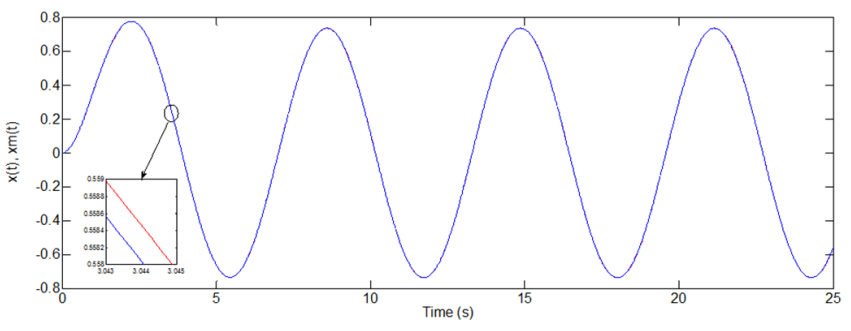

Fig. 2. Comparison of the state plant signal with reference signal

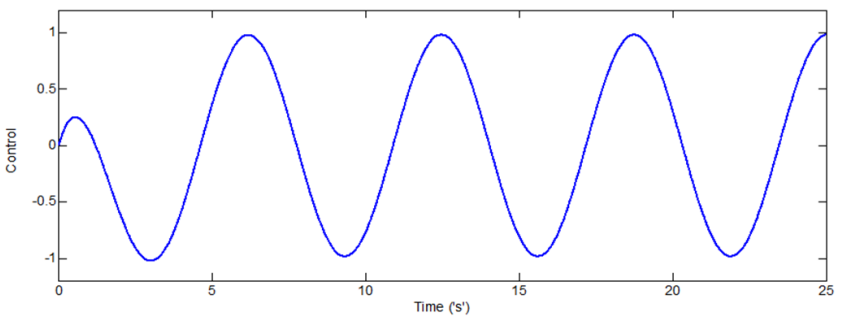

Fig. 3. Control $u(t)$

The initial conditions of the system and reference model are $y(t)=0$ and $y_{m}(t)=0$. We chose the same parameter values of the adaptive controller of the first case for this simulation, $\varphi_{1}=20$ and $\varphi_{2}=80$. The simulation results are shown in Figures 4-8. The results show the time responses of the plant state and the state of the reference model, the follow up error, and the control. Figures 6 and 7 show that the estimated parameters $a(t)$ and $b(t)$ converge to the true values of the system. Figures 2-8 indicate that the proposed method is quite efficient. The follow up error converges to zero and the control is bounded. The results are shown in Figure 4. It can be seen that $e(t) \rightarrow 0$ and $y(t) \rightarrow y_{m}(t)$ as $t \rightarrow \infty$. The convergence rate can be increased by increasing the adaptation rates $\varphi_{i}, i=1,2$, but a large value of $\varphi_{i}$ can lead to an increase in the sensitivity of the control system to noise and unmodeled dynamics that can lead to instability.

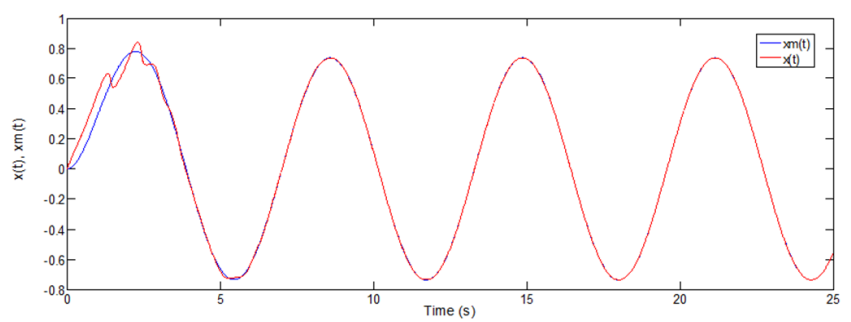

Fig. 4. State plant signal with reference signal

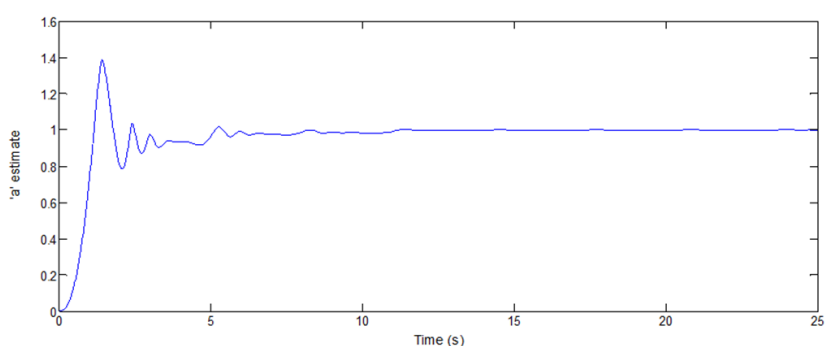

Fig. 5. Estimate of $a(t)$ 


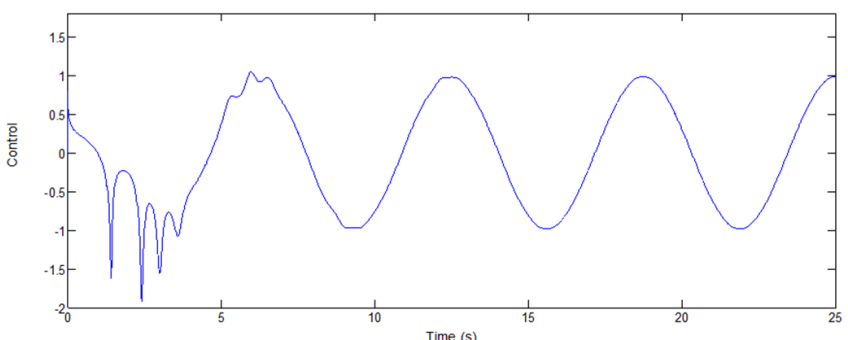

Fig. 6. Control $u(t)$

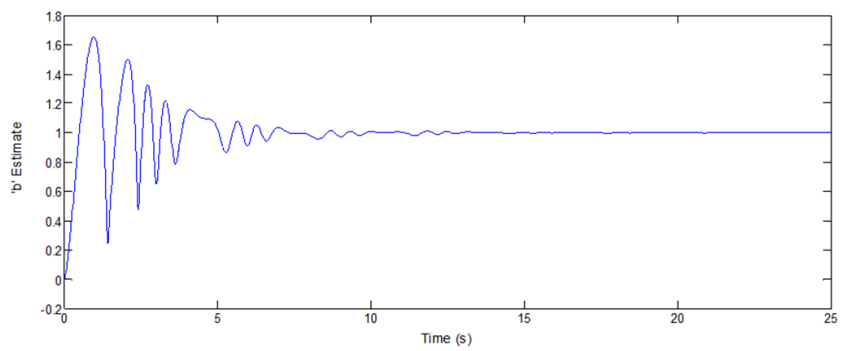

Fig. 7. Estimate of $b(t)$

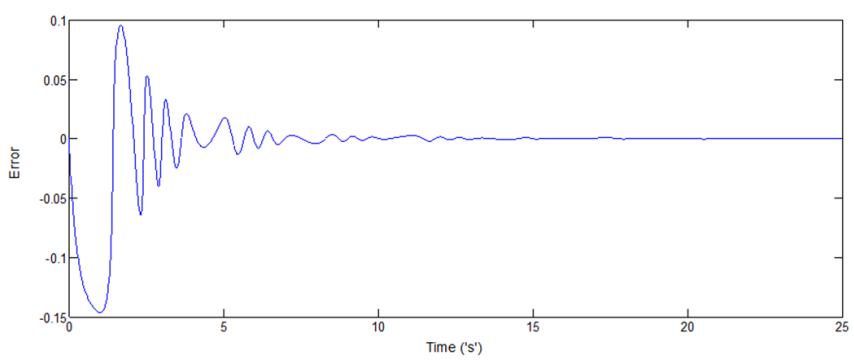

Fig. 8. Tracking error results by the proposed approach

\section{CONCLUSION}

A systematic design procedure for the design of a noninteger indirect adaptive controller for a class of non-integer systems has been presented in this paper and a new fractional indirect adaptive control scheme was presented based on a Lyapunov function. The proposed control strategy uses a MRAC configuration. It was shown that by using the proposed novel non integer adaptive controller it is possible to design a controller for the non-integer system. The effectiveness of the proposed scheme was confirmed by numerical simulations. These simulations were carried out in Matlab running in Windows 7. The step size was set to $0.001 \mathrm{~s}$, and the other settings were kept at default values. Simulation results verified the correctness of the developed theoretical approach.

\section{REFERENCES}

[1] F. A. Zaid, P. Ioannou, K. Gousman, R. Rooney, "Accommodation of failures in the f-16 aircraft using adaptive control", IEEE Control Systems Magazine, Vol. 11, No. 1, pp. 73-78, 1991

[2] B. Mirkin, P. O. Gutman, "Model reference adaptive control of state delayed system with actuator failures", International Journal of Control, Vol. 78, No. 3, pp. 186-195, 2005

[3] K. J. Astrom, B. Wittenmark, "On self-tuning regulators", Automatica, Vol. 9, No. 2, pp. 185-199, 1973
[4] P. Caines, S. Lafortune, "Adaptive control with recursive identification for stochastic linear systems", IEEE Transactions on Automatic Control, Vol. 29, No. 4, pp. 312-321, 1984

[5] G. Tao, Adaptive control design and analysis, John Wiley \& Sons, 2003

[6] P. A. Ioannou, J. Sun, Robust adaptive control, Prentice-Hall, 1996

[7] I. D. Landau, R. Lozano, M. Saad, A. Karimi, Adaptive Control, Springer, 1998

[8] M. Abedini, M. A. Nojoumian, H. Salarieh, A. Meghdari, "Model reference adaptive control in fractional order systems using discrete-time approximation methods", Communications in Nonlinear Science and Numerical Simulation, Vol. 25, No. 1-3, pp. 27-40, 2015

[9] B. Bourouba, S. Ladaci, "Comparative performance analysis of GA, $\mathrm{PSO}, \mathrm{CA}$ and $\mathrm{ABC}$ algorithms for ractional $\mathrm{PI}^{\lambda} \mathrm{D}^{\mu}$ controller tuning", 8th IEEE International Conference on Modelling, Identification and Control, Algiers, Algeria, November 15-17, 2016

[10] B. Bourouba, S. Ladaci, A. Chaabi, "Moth-Flame optimization algorithm based fractional order $\mathrm{PI}^{\lambda} \mathrm{D}^{\mu}$ controller with MRAC tuning configuration", International Journal of Systems, Control and Communications, Vol. 9, No. 2, pp. 148-171, 2018

[11] S. Ladaci, A. Charef, "On fractional adaptive control", Nonlinear Dynamics, Vol. 43, No. 4, pp. 365-378, 2006

[12] I. Podlubny, Fractional differential equations, Academic Press, 1999

[13] I. Podlubny, "Fractional-order systems and $\mathrm{PI}^{\lambda} \mathrm{D}^{\mu}$ controllers", IEEE Transactions on Automatic Control, Vol. 44, No. 1, pp. 208-214, 1999

[14] R. Hilfer, Applications of fractional calculus in physic, World Scientific, 2000

[15] B. Bourouba, S. Ladaci, A. Chaabi, "Reduced-order model approximation of fractional-order systems using differential evolution algorithm", Journal of Control, Automation and Electrical Systems, Vol. 29 , pp. 32-43, 2018

[16] P. L. Butzer, U. Westphal, An introduction to fractional calculus, World Scientific, 2000

[17] M. P. Aghababa, "Stabilization of a class of fractional-order chaotic systemsusing a non-smooth control methodology", Nonlinear Dynamics, Vol. 89, No. 3, pp. 1357-1370, 2017

[18] K. Khettab, S. Ladaci, Y. Bensafia, "Fuzzy adaptive control of a fractional order chaotic system with unknown control gain sign using a fractional order Nussbaum gain", IEEE/CAA Journal of Automatica Sinica, Vol. 6, No. 3, pp. 816-823, 2019 